\title{
Proposta de um Modelo de Avaliação de Jogos Educacionais
}

\author{
Rafael Savi* \\ Christiane Gresse von Wangenheim** \\ Vania Ulbricht ${ }^{* * *}$ \\ Tarcisio Vanzin ${ }^{* * * *}$
}

Resumo: Este artigo apresenta e propõe um modelo para a avaliação de jogos educacionais baseado no modelo de avaliação de programas de treinamento de Kirkpatrick, nas estratégias motivacionais do modelo ARCS de Keller, na área de experiência do usuário e na taxonomia de objetivos educacionais de Bloom. A estrutura do modelo e um questionário para a coleta de dados com base da percepção dos alunos são apresentados. Espera-se que este modelo possa trazer contribuições para a qualidade de jogos educacionais e a orientar professores na seleção e uso deste tipo de material educacional.

Palavras-chave: jogos educacionais; avaliação; experiência do usuário

\section{Propose of a Model for Educational Games Evaluation}

Abstract: This paper presents and proposes a model for educational games evaluation based on the Kirkpatrick's model for evaluating training programs, the motivational strategies of Keller's ARCS model, the user experience field and the taxonomy of educational objectives from Bloom. The model's structure and a questionnaire for data collection based on students perception of the games are presented. We expect that this model contributes to increase the educational games quality and helps teachers to select and use this type of educational material.

Keywords: educational games; evaluation; user experience

\section{Introdução}

Existe a expectativa entre professores de que jogos educacionais podem trazer benefícios para os processos de ensino e aprendizagem e diversos jogos já foram desenvolvidos e são utilizados em diferentes níveis de ensino e disciplinas (SAVI; ULBRICHT, 2009).

\footnotetext{
* Doutorando do Programa de Pós-Graduação em Engenharia e Gestão do Conhecimento - EGC/UFSC. rafaelsavi@gmail.com

** Dr.rer. nat., Professora do Departamento de Informática e Estatística - INE/ UFSC. gresse@gmail.com

*** Dra., Professora do Programa de Pós-Graduação em Engenharia e Gestão do Conhecimento - EGC/UFSC. ulbricht@floripa.com.br

**** Dr., Professor do Programa de Pós-Graduação em Engenharia e Gestão do Conhecimento - EGC/UFSC. tvanzin@yahoo.com.br
} 
Mas, embora se tenha indícios de que os jogos educacionais possam ser ferramentas capazes de aprimorar o processo de ensino-aprendizagem (PRENSKY, 2001; GARRIS; AHLERS; DRISKELL, 2002), e que esse tipo de recurso começa a atrair a atenção de professores e alunos, muitas vezes não se conhece direito o grau de contribuição que determinados jogos educacionais podem trazer (AKILLI, 2007).

Atualmente, a avaliação dos jogos educacionais geralmente é limitada e, por vezes, inexistente (CONNOLLY; STANSFIELD; HAINEY, 2007). Hays (2005) comenta que em muitos casos a decisão em se utilizar jogos educacionais é baseada em suposições de seus benefícios, ao invés de ser fundamentada em avaliações mais formais e concretas.

Até o momento houve poucas tentativas de se desenvolver modelos para auxiliarem as avaliações destes materiais educacionais (KIRRIEMUIR, MCFARLANE, 2004; FREITAS; OLIVER, 2006). A inexistência de um modelo que facilite a avaliação de jogos educacionais faz com que existam poucos dados para comprovar ou não os benefícios desses artefatos (FREITAS; OLIVER, 2006).

Assim, será proposto neste artigo um modelo para avaliação de jogos educacionais. $\mathrm{O}$ modelo tem seu foco em jogos que possam ser utilizados como material educacional para apoiar o processo de ensino e aprendizagem de conteúdos curriculares, ou seja, jogos com objetivos educacionais bem definidos para professores utilizarem como material de ensino em assuntos planejados nas suas disciplinas.

O modelo procura avaliar se um jogo: (i) consegue motivar os estudantes a utilizarem o recurso como material de aprendizagem; (ii) proporciona uma boa experiência nos usuários (p.ex. se ele é divertido); e (iii) se gera uma percepção de utilidade educacional entre seus usuários (ou seja, se os alunos acham que estão aprendendo com o jogo).

Um requisito importante considerado na proposta do modelo é de não consumir muito tempo na sua aplicação, já que professores normalmente não dispõem de muitas aulas que possam ser ocupadas para o teste do jogo e para longas sessões de avaliações. Outro requisito é que o modelo precisa ser sistematizado de forma a possibilitar repetidas avaliações para viabilizar comparações entre diferentes versões de um mesmo jogo, ou comparações entre jogos diferentes.

Uma pesquisa na literatura apontou alguns referenciais teóricos onde o modelo proposto neste artigo poderia se ancorar. Identificou-se, por exemplo, que o nível 1 do modelo de avaliação de treinamento de Kirkpatrick (1994), que foca-se na reação dos alunos, pode ser aplicado sem demandar muito tempo tanto na obtenção de dados como na análise. O modelo ARCS (KELLER, 2009) poderia ser tomado como base para a avaliação do nível da motivação dos alunos ao usarem os jogos. A área de experiência do usuário (User eXperience - UX) poderia ser aplicada em jogos para avaliarmos se um jogo é divertido e proporciona uma experiência agradável aos alunos. E a percepção de impacto na aprendizagem poderia ser feita com apoio da taxonomia de Bloom (BLOOM, 1956; CHAPMAN, 2009).

O modelo aqui proposto e as suas âncoras teóricas são detalhados nas seções a seguir.

\section{O Modelo de avaliação de treinamentos de Kirkpatrick}

Donald Kirkpatrick (1994) criou um modelo de avaliação de treinamento baseado em quatro níveis. Segundo Kirckpatrick, cada nível tem sua importância e, conforme se passa de um nível para outro seguinte, o processo ser torna mais complexo e demorado, 
porém fornece resultados mais valiosos. Os níveis são: (1) Reação: onde se mede a satisfação e valor percebido do treinamento pelos participantes; (2) Aprendizagem: levanta o quanto os participantes podem mudar de atitude, ampliar seus conhecimentos e/ou habilidades; (3) Comportamento: identifica o quanto os participantes mudaram seu comportamento em decorrência do que foi aprendido. (4) Resultados: identifica os ganhos obtidos com o treinamento.

O modelo de Kirkpatrick é um dos modelos de avaliação mais conhecidos e utilizados (FOXON, 1989).

O modelo de avaliação proposto neste artigo tem como base o nível 1 de Kirkpatrick e é focado na reação dos alunos ao utilizarem um jogo educacional. Para Kirkpatrick, avaliar a reação é como medir a satisfação de um cliente. Se um treinamento deve ser efetivo, é importante que os alunos reajam favoravelmente a ele, do contrário não se sentirão motivados para aprender. Complementa ainda que a avaliação da reação é importante porque ajuda a indicar o quanto eficaz um programa de treinamento foi e aponta formas de melhorá-lo.

O nível 1 de Kirkpatrick (reação) avalia qual foi a percepção do participantes a respeito da experiência de aprendizagem. Para isso utiliza como ferramenta de avaliação formulários de feedback, pesquisas após o treinamento ou questionários. Geralmente é rápido e fácil de obter e tem baixo custo tanto para obter como para analisar (CHAPMAN, 2009b).

\section{O MODELO ARCS}

Motivação para aprender é um elemento essencial em qualquer sistema educacional (KELLER, 2009), por isso os ambientes de aprendizagem precisam ser projetados com cuidado para provocarem um nível adequado de motivação nos estudantes (HUANG; HUANG; TSCHOPP, 2010).

A motivação no contexto educacional é definido por Maehr (1976) como um engajamento voluntário em continuar a aprender mais sobre determinado assunto (KELLER, J. M.; SUZUKI, K).

Um dos métodos existentes que objetiva empregar estratégias motivacionais no projeto de materiais instrucionais é o modelo ARCS de John Keller (DEMPSEY; JOHNSON, 1998). O modelo ARCS tem seu foco na interação dos alunos com os ambientes de aprendizagem e é derivado da teoria expectativa-valor (KELLER, 2009). Esta teoria aponta que a expectativa (que está ligada a uma probabilidade subjetiva de um indivíduo obter sucesso) e valores (que estão ligados a satisfação de necessidades pessoais ou motivos) são determinantes chave do esforço empregado em uma atividade (DEMPSEY; JOHNSON, 1998; KELLER, 2009).

ARCS é um acrônimo que identifica quatro categorias de estratégias importantes para que se consiga motivar os alunos na aprendizagem: Atenção, Relevância, Confiança e Satisfação (Attention, Relevance, Confidence, Satisfaction)(KELLER, 2009).

Atenção - Refere-se às respostas congnitivas dos alunos aos estímulos instrucionais (HUANG; HUANG; TSCHOPP, 2010). A atenção é um elemento motivacional e também pré-requisito para aprendizagem. O desafio é obter e manter um nível satisfatório da atenção dos alunos ao longo de um período de aprendizagem (KELLER, 2009). 
Relevância - Embora necessários, a atenção e curiosidade do aluno não são condições suficientes para a motivação aos estudos. O aluno também precisa perceber que a proposta educacional seja consistente com seus objetivos, que ele consiga conectar o conteúdo da aprendizagem com seu futuro profissional ou acadêmico. Quantas vezes vemos estudantes perguntarem "porque tenho que estudar isso?". Caso não se apresente uma boa resposta, estamos diante de um problema de relevância (KELLER, 2009). Também representa o nível de associação que os alunos conseguem perceber entre seus conhecimentos prévios e as novas informações (HUANG; HUANG; TSCHOPP, 2010).

Confiança - A terceira estratégia está relacionada em criar expectativas positivas aos estudantes. Isso pode ser alcançado ao se proporcionar experiências de sucesso decorrentes de suas próprias habilidades e esforço. Este fator tem influência na persistência dos estudantes (KELLER, 2009; HUANG; HUANG; TSCHOPP, 2010).

Satisfação - Os alunos precisam ter sentimentos positivos sobre a experiência de aprendizagem, e isso pode vir com recompensas e reconhecimento. Também recomenda-se providenciar tão cedo quanto possível oportunidades para os alunos aplicarem o que foi aprendido. Os estudantes devem sentir que o esforço aos estudos foi apropriado e que houve consistência entre objetivos, conteúdo e testes (KELLER, 2009; HUANG; HUANG; TSCHOPP, 2010).

O modelo ARCS é uma framework que pode ser usado tanto para o desenvolvimento de estratégias motivacionais (a serem incluídas na produção de novos materiais educacionais), como também para medir o nível de motivação de materiais educacionais existentes (DEMPSEY; JOHNSON, 1998; HUANG; HUANG; TSCHOPP, 2010).

Para ser utilizado na avaliação de materiais, o próprio Keller desenvolveu um instrumento denominado Instructional Materials Motivational Scale (IMMS) (DEMPSEY; JOHNSON, 1998), que consiste em um questionário para ser aplicado depois que os estudantes utilizam um material educacional.

O modelo ARCS vem sendo utilizado em diversos estudos para avaliar a motivação dos estudantes ao utilizarem materiais educacionais, já foi validade para uso em ambientes interativos, e alguns estudos já aplicaram o modelo no contexto de jogos educacionais (HUANG; HUANG; TSCHOPP, 2010).

Astleitner \& Wiesner (2004) apontam que, claramente, o modelo ARCS pode ser aplicado para se investigar aspectos motivacionais de jogos educacionais.

\section{USER EXPERIENCE EM JOGOS}

Embora não exista consenso ou uma definição unificada sobre experiência do usuário (User eXperience - UX), algumas tentativas têm sido feitas. A ISO 9241-210:2008 considera que a área de UX se concentra na percepção e resposta de uma pessoa sobre o uso de um produto, sistema ou serviço (TAKATALO; HÄKKINEN; KAISTINEN, 2010).

$\mathrm{Na}$ área de UX os produtos não são vistos primariamente como um pacote de funcionalidades e benefícios - eles provocam experiências. E é objetivo da UX avaliar e ampliar o entendimento dessas experiências que as pessoas têm com os produtos. 
Essas experiências, decorrentes da interação com produtos, sistemas ou serviços, podem gerar mudanças no estado emocional das pessoas (GÁMEZ, 2009). Com isso, a avaliação da experiência pode ser feita ao se avaliar os elementos presentes num processo de interação (GÁMEZ, 2009).

A UX contempla a interação do indivíduo com o produto por inteiro, considerando também pensamentos, sentimentos, prazer e demais percepções que resultam da interação (TULLIS; ALBERT, 2008). A UX procura compreender tudo aquilo que o usuário fala ou pensa sobre um produto, como por exemplo, ele pode reportar que foi fácil de usar, que foi confuso, ou que ele excedeu suas expectativas (TULLIS; ALBERT, 2008).

Existe uma expectativa de que a utilização de jogos produza experiências positivas (GÁMEZ, 2009). Os jogadores interagem com outros objetos, se envolvem e se aprofundam no ambiente do jogo, e isso vem acompanhado de emoções - que são consideradas parte fundamental do uso de jogos (TAKATALO; HÄKKINEN; KAISTINEN, 2010).

Mas como fazer para se avaliar a experiência do usuário em jogos? Quatro modelos foram encontrados na literatura (POELS; KORT; IJSSELSTEIJN, 2007; JENNETT, 2008; FU; SU;YU, 2009; GÁMEZ, 2009), e todos convergem no sentido de considerar o conceito de "experiência" difícil de ser descrito e assimilado. Então, buscam entender a experiência do usuário em jogos a partir dos elementos de interação que a constituem, como por exemplo, diversão, imersão, desafio, etc. No entanto, não existe entre estes modelos consenso sobre quais são estes elementos, e cada um deles faz uma proposta diferente. Porém, uma análise inicial mostrou que existiam alguns conceitos que se repetiam nestes modelos e partiu-se para uma análise mais detalhada para identificar os elementos mais frequentemente utilizados para descrever a experiência do usuário em jogos. Os conceitos que se repetiram em pelo menos 3 modelos foram considerados como os mais consolidados e selecionados para uso no modelo de avaliação de jogos educacionais proposto neste artigo. A seguir estes conceitos são listados e detalhados.

1. Atenção, fluxo, IMERSÃO: Bons jogos levam o jogador a ter uma experiência de profundo envolvimento no jogo, que geralmente provoca um desvio de foco do mundo real para o mundo do jogo. Isso resulta num esquecimento das preocupações, levando a depoimentos tais como "minha visão estava totalmente engajada no mundo do jogo" e "eu não estava percebendo meu mundo real" (TAKATALO; HÄKKINEN; KAISTINEN, 2010). A imersão provoca uma distorção da noção de tempo, sendo que muitos jogadores relatam que passam noites ou finais de semana jogando sem perceberem o tempo passar (TAKATALO; HÄKKINEN; KAISTINEN, 2010).

2. Co-presença, presença social, INTERACÃO SOCIAL: O envolvimento com outras pessoas é um elemento de diversão nos jogos, e está relacionado com o sentimento de compartilhar um ambiente com outras pessoas e de se ter um papel ativo nele. Decorrente disso surgem sentimentos de diversão em grupo, de conexão com outras pessoas, cooperação, conquistas dentro de grupos e de união. Para possibilitar a interação social os jogos devem criar oportunidades para os jogadores competirem, cooperarem e se conectarem. As pessoas gostam de interagir com outras pessoas, passar um tempo com amigos, assistir os outros jogarem, conversar 
sobre o jogo, e observar as expressões e reações dos outros enquanto disputam, ganham ou perdem (TAKATALO; HÄKKINEN; KAISTINEN, 2010).

3. DESAFIO, suspense: Desafio é um dos aspectos mais importantes nos bons jogos (SWEETSER; WYETH, 2005). Um jogo precisa ser suficientemente desafiador, estar adequado ao nível de habilidade do jogador, apresentar variações no nível de dificuldade, e manter um ritmo adequado. O ritmo é um componente importante de desafio, pois a velocidade em que novos detalhes ou desafios são revelados ao jogador pode ser programada para manter um nível apropriado de tensão e desafio ao longo do jogo.Novos obstáculos, situações e variações de atividades devem ser planejadas ao longo do jogo para minimizar a fadiga do jogador e oferecer uma experiência que mantenha sua vontade de continuar jogando. É necessário equilibrar as habilidades de uma pessoa com os desafios associados a uma atividade, pois quando o desafio é maior do que a habilidade tem-se como resultado a ansiedade do jogador, e quando a habilidade é maior do que o desafio tem-se como resultado a apatia. O nível de dificuldade deve variar e aumentar gradualmente para manter o interesse do jogador na medida em que ele desenvolve suas habilidades. $\mathrm{O}$ desafio dos jogos traz sentimentos de suspense, tensão, pressão, expectativa, ansiedade e de estímulo. Quando desafios são superados o jogador sente alívio, realização e euforia. A satisfação do usuário vem ao se completar tarefas difíceis, derrotar oponentes, testar habilidades, desenvolver habilidades, alcançar uma meta desejada, e ao confrontar o perigo (POELS; KORT; IJSSELSTEIJN, 2007; TAKATALO; HÄKKINEN; KAISTINEN, 2010).

4. DIVERSÃO: Os jogos devem proporcionar sentimentos de diversão, prazer, relaxamento, distração e satisfação (POELS; KORT; IJSSELSTEIJN, 2007). Quando jogar for algo especial para o jogador haverá uma experiência fortemente positiva, acompanhada do desejo de voltar a participar do jogo e de recomendá-lo para colegas (TAKATALO; HÄKKINEN; KAISTINEN, 2010).

5. CONTROLE: Está relacionado com a sensação de independência, domínio (TAKATALO; HÄKKINEN; KAISTINEN, 2010) autonomia, poder e liberdade (POELS; KORT; IJSSELSTEIJN, 2007). Os jogadores devem poder exercer um senso de controle sobre suas ações, conseguir traduzir suas intenções em comportamentos dentro do jogo, sentir controle sobre os movimentos de seus personagem e a maneira como ele explora o ambiente. A movimentação dos personagem deve ocorrer eficientemente no ambiente do jogo, e a manipulação de objetos deve acontecer com facilidade. O jogador deve ter um senso de controle sobre a interface e controles do jogo, que devem ser fáceis de aprender, para então explorar o jogo com liberdade e no seu próprio ritmo (TAKATALO; HÄKKINEN; KAISTINEN, 2010).

6. COMPETÊNCIA, habilidades do jogador: A competência é uma medida combinada de habilidades do jogador e sentimentos positivos de eficiência. "Eu senti que poderia atender as demandas das situações do jogo" (TAKATALO; HÄKKINEN; KAISTINEN, 2010). Está relacionada com a percepção de habilidades, controle e uso dessas habilidades para explorar o jogo e progredir 
(POELS; KORT; IJSSELSTEIJN, 2007). Para proporcionarem uma boa experiência os jogos devem apoiar o desenvolvimento e domínio de habilidades do jogador. $\mathrm{O}$ jogador precisa perceber que suas habilidades estão num nível em que é possível superar os desafios do jogo. Como a dificuldade dos desafios tende a aumentar, será necessário que o jogador consiga desenvolver suas habilidades para avançar no jogo e se divertir. O jogo não pode demandar habilidades especiais do jogador. Devem haver informações suficientes para que se comece a jogar sem a necessidade da leitura de longos manuais e sem a demanda por longas explicações sobre regras, cenário ou história do jogo - que podem chatear o jogador (TAKATALO; HÄKKINEN; KAISTINEN, 2010).

Estes são os conceitos adotados para compor a experiência do usuário de jogos no modelo proposto neste artigo.

\section{Taxonomia de Bloom}

A taxonomia de Bloom (BLOOM, 1956) foi criada dentro de um contexto acadêmico na década de 1950 com o objetivo de apoiar os processos de projeto e avaliação educacional (CHAPMAN, 2009b). A parte do trabalho de Bloom que ficou mais conhecida é a "Taxonomia e Objetivos no Domínio Cognitivo".

Benjamin Bloom criou, em conjunto com outros pesquisadores, uma classificação de níveis de raciocínio em seis níveis numa sequência que vai do mais simples (conhecimento) ao mais complexo (avaliação) (BARATO, 2009).

É uma estrutura que pode ser aplicada para planejar, projetar e avaliar a efetividade da aprendizagem e de treinamentos (CHAPMAN, 2009b).

A seguir são apresentadas as categorias criadas pelo grupo de Bloom e as formas de descrever os conhecimentos que se deseja que os alunos desenvolvam (WALL; TELLES, 2004).

- Conhecimento - lembrar informações sobre: fatos, datas, palavras, teorias, métodos, classificações, lugares, regras, critérios, procedimentos etc.

- Compreensão - entender a informação ou o fato, captar seu significado, utilizála em contextos diferentes.

- Aplicação - aplicar o conhecimento em situações concretas

- Análise - identificar as partes e suas inter-relações

- Síntese - combinar partes não organizadas para formar um todo

- Avaliação - julgar o valor do conhecimento

No modelo de avaliação aqui proposto, os três primeiros níveis da taxonomia de Bloom serão levados em consideração - conhecimento, compreensão e aplicação. Os três outros níveis são considerados de maior complexidade e não serão abordados. A estratégia para o modelo de avaliação de jogos educacionais será obter a percepção do aluno (Kirkpatrick nível 1) em relação a sua capacidade de lembrar, entender e aplicar (três primeiros níveis da taxonomia de Bloom) os assuntos abordados em um determinado jogo. 


\section{Estrutura do Modelo de Avaliação Proposto}

A figura 1 a seguir apresenta a estrutura do modelo de avaliação de jogos educacionais proposto neste artigo e seus referenciais teóricos. Esta estrutura destaca no primeiro círculo a característica do modelo buscar avaliar a reação dos alunos, ou seja, a percepção deles em relação a experiência de aprendizagem, conforme o nível 1 de Kirkpatrick, ao utilizarem um determinado jogo educacional.

Mas o modelo de kirkpatrick deixa em aberto os parâmetros que devem ser avaliados. Considerando esta proposta de se avaliar um jogo através da percepção que os alunos tiveram, foram incluídos nesta estrutura o modelo ARCS para avaliação do nível de motivação, componentes de user experience em jogos para avaliação da experiência de interação com o jogo, e princípios da taxonomia de Bloom para avaliação do impacto na aprendizagem do aluno.

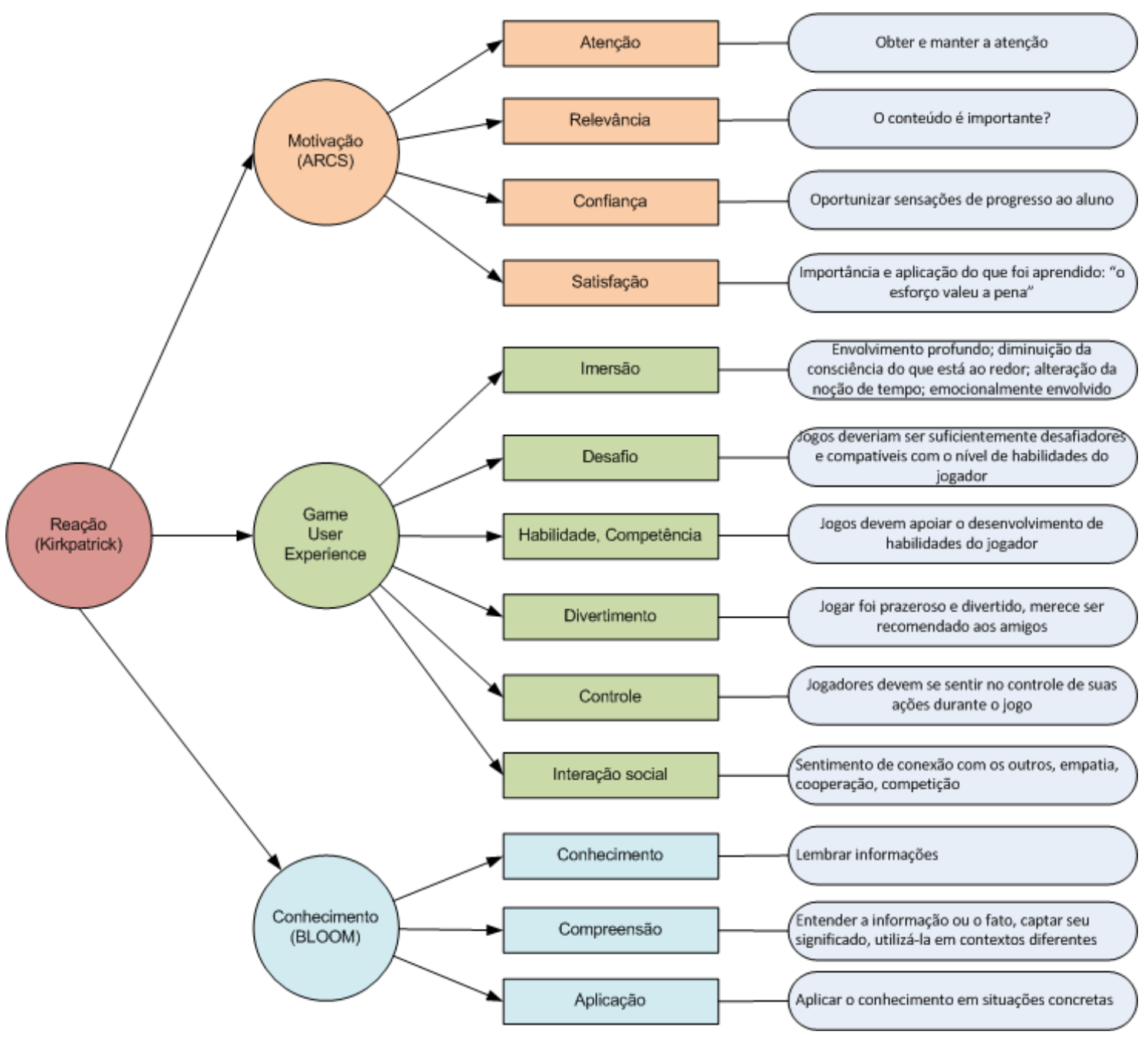

Figura 1- Modelo de avaliação de jogos educacionais

Como a avaliação destes três aspectos deve ser obtida a partir da percepção dos alunos, foi desenvolvido um questionário para coletar dados sobre os parâmetros que formam este modelo de avaliação. O questionário é apresentado a seguir, possui itens sobre 
variados aspectos dos parâmetros do modelo de avaliação, e são apresentados na forma de afirmações para os alunos indicarem o quanto concordam com elas de acordo com uma escala Likert de 7 pontos variando de "discordo fortemente" até "concordo fortemente". As questões para avaliação da motivação foram obtidas do estudo de Huang, Huang e Tschopp (2010) que indicaram uma versão adaptada para jogos do questionário Instructional Materials Motivational Survey - IMMS, que originalmente foi proposto por Keller (HUANG et al, 2006). As questões de experiência do usuário vieram de uma seleção de questionários sobre user experience em jogos (JENETT, et al, 2008; FU; SU; YU, 2009; GÁMEZ, 2009), e as questões sobre impacto na aprendizagem são perguntas diretas sobre os três primeiros níveis da taxonomia de Bloom.

\section{Quadro 1 - Questionário para avaliação de jogos educacionais}

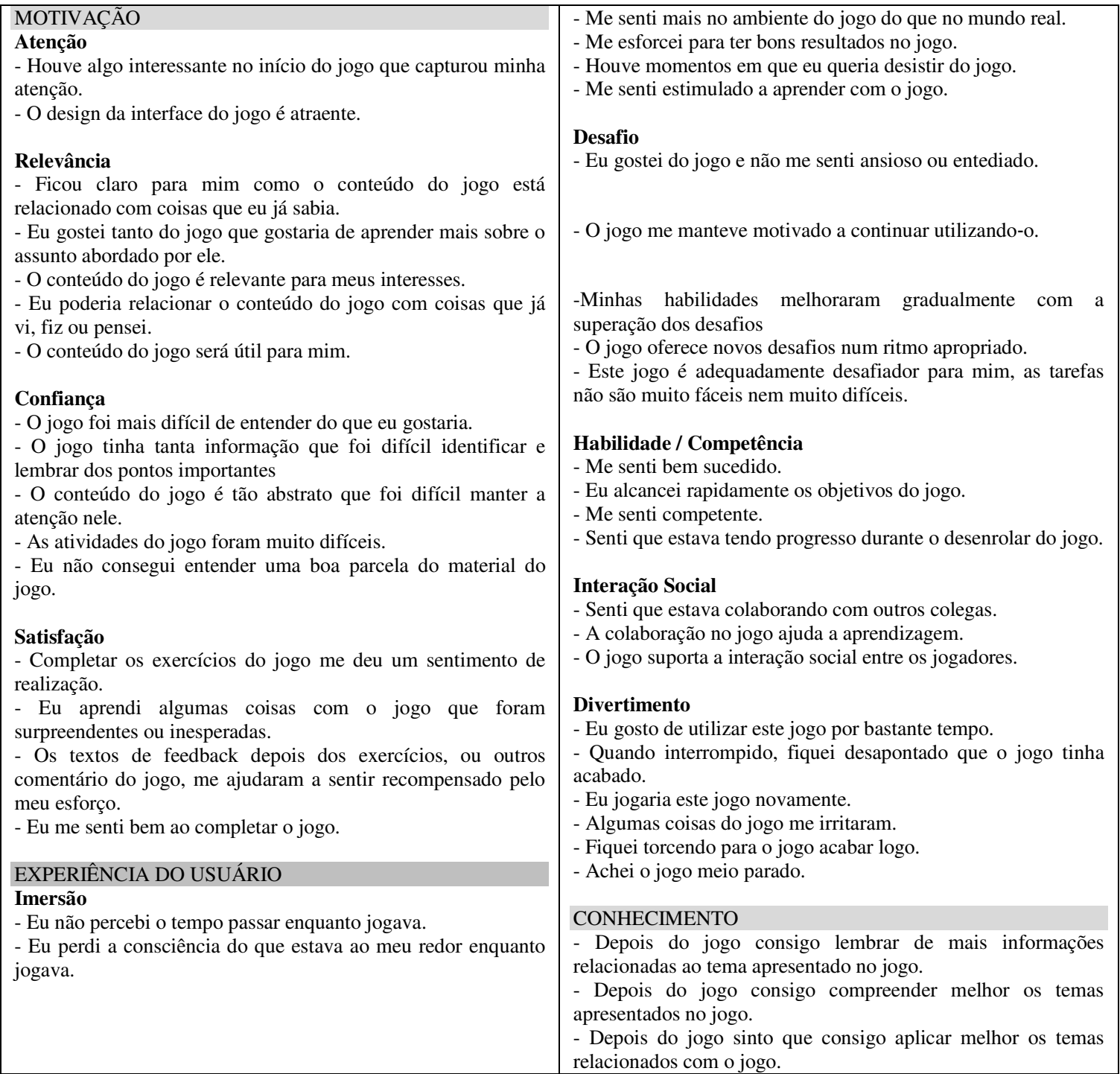

Depois da coleta dos dados pelo questionário, a análise oferece como resultado uma pontuação sobre diferentes aspectos a respeito da avaliação de um jogo educacional. Tal resultado pode trazer diversas contribuições, tais como oportunidades de melhoria em jogos (tanto em jogos finalizados como em jogos ainda em versão de desenvolvimento), subsídios para professores decidirem entre um conjunto de jogos semelhantes aquele 
que trás melhores resultados para a turma de alunos, ou mesmo argumentos que apontem tendências de aprovação ou não de uma turma sobre o uso de um jogo educacional.

\section{Considerações Finais}

Atualmente há uma carência por modelos que possam ser utilizados para a avaliação de jogos educacionais.

A avaliação de material educacional é uma atividade reconhecida como essencial no processo de ensino e aprendizagem, pois é preciso avaliar se um produto educacional consegue cumprir aquilo que foi planejado para ele (MONTILVA; BARRIOS; SANDIA, 2002; PADRÓN; DÍAZ; AEDO, 2007). Isto é importante porque mesmo os designers instrucionais experientes não desenvolvem materiais perfeitos, que na prática podem não funcionar da maneira que foram planejados (MORRISON; ROSS; KEMP, 2001; ROMISZOWSKI, 2004).

Padrón, Díaz, e Aedo (2007) ressaltam que a avaliação subsidia os desenvolvedores com informações sobre a utilidade do material. Mas apesar de sua importância, a avaliação é negligenciada na criação de muitos materiais didáticos (PADRÓN; DÍAZ; AEDO, 2007). Isto é preocupante porque o pouco uso de avaliação no projeto de materiais educacionais pode fazer com que não se perceba os problemas, dando uma falsa impressão de sucesso (ROMISZOWSKI, 2004).

Este artigo apresentou uma proposta de modelo de avaliação de jogos educacionais que busca obter a percepção dos alunos sobre o nível de motivação proporcionado pelo jogo, a experiência de interação e impacto na aprendizagem. Espera-se que o uso deste modelo possa trazer contribuições para a qualidade de jogos e a orientar professores no uso deste tipo de material. No entanto, é necessário salientar que agora esta proposta precisa ser validada na prática e entrará numa fase de aplicação e testes.

\section{Referências}

AKILLI, G. K. Games and Simulations: A new approach in education. In: Gibson D.; Aldrich C.; Prensky M. Games and simulations in online learning: research and development frameworks. Information Science Publishing, Hershey/PA, p. 1-20, 2007.

ASTLEITNER, H.; WIESNER, C. An Integrated Model of Multimedia Learning and Motivation.

Journal of Educational Multimedia and Hypermedia, , v. 13, n. 1, p. 3-21, 2004.

BARATO, Jarbas. Taxonomia de Bloom. 2009. Disponível em:

<http://jarbas.wordpress.com/2009/11/21/taxonomia-de-bloom/ >. Acesso em: 16 Mar. 2010.

BLOOM, B.S. Taxonomy of educational objectives: The classification of educational goals: Handbook I, cognitive domain. New York; Toronto: Longmans, Green. 1956. 207 p.

CHAPMAN, Alan. Bloom's taxonomy - learning domains. Businessballs, 2009. Disponível em: $<$ http://www.businessballs.com/bloomstaxonomyoflearningdomains.htm>. Acesso em: fev. 2010.

CHAPMAN, Alan. Kirkpatrick's learning and training evaluation theory. Businessballs, 2009. Disponível em: <http://www.businessballs.com/kirkpatricklearningevaluationmodel.htm>. Acesso em: fev. 2010.

CONNOLLY, T. M.; STANSFIELD, M.; HAINEY, T. An application of games-based learning within software engineering. British Journal of Educational Technology, v. 38, p. 416-428, Maio 2007.

DEMPSEY, J. V.; JOHNSON, R. B. The development of an ARCS gaming scale. Journal of Instructional Psychology, v. 25, n. 4. p 215-221, 1998. 
FOXON, M. Evaluation of Training and Development Programs: A Review of the Literature. Australian Journal of Educational Technology 5(2): p. 89-104, 1989.

FREITAS, S. D.; OLIVER, M. How can exploratory learning with games and simulations within the curriculum be most effectively evaluated? Comput. Educ., , v. 46, n. 3, p. 249-264, 2006.

FU, F.; SU, R.; YU, S. EGameFlow: A scale to measure learners' enjoyment of e-learning games.

Comput. Educ., , v. 52, n. 1, p. 101-112, 2009.

GÁMEZ, E. H. C. On the Core Elements of the Experience of Playing Video Games.2009. 208 f. Tese de doutorado - UCL Interaction Centre Department of Computer Science, 2009.

GARRIS, R.; AHLERS, R.; DRISKELL, J. E. Games, Motivation, and Learning: A Research and Practice Model. Simulation Gaming, v. 33, n. 4, p. 441-467, 2002.

HAYS, R.T. The Effectiveness of Instructional Games: A Literature Review and Discussion. Orlando: Naval Air Warfare Center Training System Division, 2005.

HUANG, W. et al. A Preliminary Validation of Attention, Relevance, Confidence and Satisfaction Model-Based Instructional Material Motivational Survey in a Computer-Based Tutorial Setting. British Journal of Educational Technology, v. 37, n. 2, p. 243-259, Março, 2006.

HUANG, W.; HUANG, W.; TSCHOPP, J. Sustaining iterative game playing processes in DGBL: The relationship between motivational processing and outcome processing. Comput. Educ., v. 55, n. 2, p. 789-797, 2010.

JENNETT, C. et al. Measuring and defining the experience of immersion in games. Int. J. Hum.Comput. Stud., , v. 66, n. 9, p. 641-661, 2008.

KIRKPATRICK, Donald L., Evaluating Training Programs - The Four Levels. Berrett-Koehler Publishers, Inc. 1994.

KELLER, J. M.; SUZUKI, K. Learner Motivation and E-Learning Design: A Multinationally Validated Process. Journal of Educational Media, v. 29, n. 3, p. 229-239, Outubro, 2004.

KELLER, J. M. Motivational Design for Learning and Performance: The ARCS Model Approach. Springer, 2009.

KIRRIEMUIR, John; MCFARLANE, Angela. Literature Review in Games and Learning. Bristol: Futurelab, 2004. 39 p.

MAEHR, M. L. Continuing Motivation: An Analysis of a Seldom Considered Educational Outcome. Review of Educational Research, , v. 46, n. 3, p. 443 -462, Summer 1976.

MONTILVA, J.; BARRIOS, J.; SANDIA, B. A Method Engineering Approach to Evaluate Instructional Products. Actas de la XXVIII Conferencia Latinoamericana de Informática - CLEI'2002.

Montevidéu, Uruguai, Novembro, 2002.

MORRISON, G. R.; ROSS, S. M.; KEMP, J. E. Designing Effective Instruction, 4th Edition. 4 . Wiley, 2003.

PADRÓN, C.; DÍAZ, P.; AEDO, I. Towards an Effective Evaluation Framework for IMS LD-Based Didactic Materials: Criteria and Measures. In: HUMAN-COMPUTER INTERACTION APPLICATIONS AND SERVICES, 2007, p.312-321.

POELS, K.; KORT, Y. D.; IJSSELSTEIJN, W. "It is always a lot of fun!": exploring dimensions of digital game experience using focus group methodology In: PROCEEDINGS OF THE 2007 CONFERENCE ON FUTURE PLAY. Toronto, Canada: ACM, 2007.p.83-89.

PRENSKY, Mark. Digital game-based learning. New York: McGraw-Hill, 2001.

ROMISZOWSKI, H. P. Avaliação no Design Instrucional e Qualidade da Educação a Distância: qual a relação? Revista Brasileira de Aprendizagem Aberta e a Distância, v. 03, 2004. 
SAVI, R.; ULBRICHT, V. R. Jogos Digitais Educacionais: Benefícios e Desafios. RENOTE - Revista Novas Tecnologias na Educação, v. 6, p. 1-10, 2008.

SWEETSER, P.; WYETH, P. GameFlow: a model for evaluating player enjoyment in games. Comput. Entertain., , v. 3, n. 3, p. 3-3, 2005.

TAKATALO, J.; HÄKKINEN, J.; KAISTINEN, J.; Nyman, G. Presence, Involvement, and Flow in Digital Games. In: BERNHAUPT, R. Evaluating User Experience in Games: Concepts and Methods. Springer, 2010, p, 23-46.

TULLIS, T.; ALBERT, W. Measuring the User Experience: Collecting, Analyzing, and Presenting Usability Metrics. Morgan Kaufmann, 2008.

WALL, P. TELLES, M. A Taxonomia de Bloom. Dynamiclab, 2004. Disponível em:<http://www.dynamiclab.com/moodle/mod/forum/discuss.php?d=436>. Acesso em: 19 out. 2010. 